\title{
Surface Properties of Polypropylene Films as Biomaterials
}

\author{
Nobuhiko YUI,* Yorito SUZUKI, Hideharu MoRI, and Minoru TeRANo* \\ School of Materials Science, Japan Advanced Institute of Science and Technology, \\ 15 Asahidai, Tatsunokuchi, Ishikawa 923-12, Japan
}

(Received December 13, 1994)

\begin{abstract}
Surface properties of polypropylene films were investigated as the preliminary study on the design of blood compatible polyolefin surfaces. It was observed that the surface crystallinity of polypropylene films was well correlated with bulk crystallinity. Smaller contact angles of polypropylene films appeared with higher surface crystallinity, which indicates that crystalline regions have higher surface free energy than amorphous regions on these surfaces. Minimum value in both albumin and fibrinogen adsorption was observed on polypropylene surfaces with a particular surface crystallinity $(c a .50 \%)$. The control of surface crystallinity including the size and distribution of crystalline and amorphous regions can dominate the fate of plasma protein adsorption. Thus, it is suggested that surface crystallinity is one of the dominant factors in the design of blood compatible polyolefin surfaces.

KEY WORDS Polypropylene / Surface Crystallinity / Surface Wettability / Protein Adsorption / Albumin / Fibrinogen / Biomaterials /
\end{abstract}

Development of excellent blood-contacting devices has required the design of nonthrombogenic polymers with high reliability. Thrombogenesis is initiated via activation pathways of both platelets and coagulation factors. Interaction between platelets and polymer surfaces has been extensively studied in terms of platelet adsorption/adhesion, and plasma protein adsorption/desorption with their dynamics. In our previous studies, it was demonstrated that the surface crystallinity of aliphatic polyamides is closely connected with platelet adhesion in vitro. ${ }^{1}$ Crystallinity of polyamides is one of the important factors for designing nonthrombogenic polymers. Semicrystalline polymers undergo very limited crystallization, which results in the formation of microstructures composed of ordered crystalline and disordered amorphous phases. Thus, this type of microstructure can affect the fate of platelets on these polymer surfaces. From these perspectives, synthesis, characterization, and blood-contacting prop-

\footnotetext{
* To whom correspondence should be addressed.
}

erties of multi-block copolymers consisting of amorphous polyether and semicrystalline polyamide segments have been thoroughly investigated in the last decade. ${ }^{1-3} \mathrm{~A}$ representative of such nonthrombogenic polymers is a series of multi-block copolymers composed of poly(propylene oxide) and nylon 610 segments.

Our systematic studies demonstrated that platelet adhesion and activation in vitro and thrombogenicity in vivo on these multi-block copolymer surfaces are greatly reduced for copolymers having a crystallite thickness of $6.0-6.5 \mathrm{~nm}$ and a long period of $12-13 \mathrm{~nm} .^{2,3}$ This suggests that the particular size and distribution of the crystalline and amorphous phases in the copolymer could be a dominant factor for preventing platelet adhesion on the copolymer surface. The control of crystallineamorphous microstructure could lead to excellent nonthrombogenic polymers. Therefore, it has been proposed that the prevention of contact-induced activation of platelets on 
crystalline-amorphous microdomain-structured polymer surfaces is critical to the design of improved nonthrombogenic polymers.

Alternative polymers which form semicrystalline states are polyolefins. Since Ziegler and Natta developed catalysts for the synthesis of polyolefins during the 1950's, polyethylene, polypropylene, and their copolymers have been widely investigated, and now have great markets in plastic industries. ${ }^{4,5}$ Although some polyolefins have been applied for disposable medical devices such as catheters, blood bags, and some extracorporeal devices, less attention has been paid to their blood compatibility. ${ }^{6}$ Thus, one question may be, is it possible to achieve blood compatible polyolefin surfaces by controlling microstructures composed of crystalline and amorphous phases? This study deals with surface properties of several polypropylene films as the preliminary study on blood compatible polyolefin surfaces. Films of polypropylenes and poly(propylene-coethylene)s with variation in semicrystalline states were characterized by means of smallangle X-ray scattering, attenuated total reflection -Fourier-transform infrared spectroscopy, surface wettability, and plasma protein adsorption.

\section{EXPERIMENTAL}

\section{Polymers}

Three grades of polypropylenes including homopolymers (PP), random-copolymers with ethylene (EPR), and block-type copolymers with ethylene (EPB) were used in this study as melt-moulding films, which were supplied from Chisso Co., Tokyo, Japan. Crystallinity of these polymers was varied by changing the polymerization conditions and copolymerization with ethylene. The weight-average molecular weights of these polypropylenes were $1.9 \times 10^{5}-3.1 \times 10^{5}$. Each polypropylene film contained Irganox ${ }^{\circledR} 1010$ (pentaerytritol-tetrakis[3-(3,5-di-tert-butyl-4-hydroxyphenyl) propionate]) as a nondiscoloring stabilizer (0.05 $w t \%)$. No remover was used in this moulding process. These polypropylene films were used after washing with ethanol and dried in vacuo. Polystyrene (PSt) as a reference sample was prepared by radical polymerization in toluene at $60^{\circ} \mathrm{C}$ using azobisisobutyronitrile as an initiator.

\section{Small-Angle X-Ray Scattering}

Small-angle X-ray scattering (SAXS) with nickel-filtered $\mathrm{Cu}-K_{\alpha}$ radiation $(\lambda=0.1542 \mathrm{~nm})$ at $50 \mathrm{kV}$ and $150 \mathrm{~mA}$ was recorded photographically using a diffractometer $(8200 \mathrm{~T}$, JEOL, Tokyo, Japan). Exposure times of $20 \mathrm{~s}$ were sufficient to produce excellent SAXS pattern. The long period was evaluated from the diameter of the SAXS pattern applying Bragg's law.

\section{Attenuated Total Reflection Fourier-Transform Infrared Spectroscopy}

Surface crystallinity of polypropylene films was characterized by attenuated total reflection Fourier-transform infrared spectroscopy (ATR-FT-IR) using a Fourier-transform infrared spectrophotometer (VALOR-III, JASCO, Tokyo, Japan) equipped with an ATR apparatus (ATR-500/M, JASCO, Tokyo, Japan). A polypropylene film was directly mounted on a Ge prism of ATR apparatus for measurement. Surface crystallinity of polypropylene films was calculated from the following equation according to the previous report. ${ }^{7}$

$$
\text { Surface crystallinity }=109 R-31.4
$$

where $R$ is the ratio of absorbance difference calculated as follows:

$$
\begin{aligned}
R= & {\left[D\left(997 \mathrm{~cm}^{-1}\right)-D\left(917 \mathrm{~cm}^{-1}\right)\right] / } \\
& {\left[D\left(972 \mathrm{~cm}^{-1}\right)-D\left(917 \mathrm{~cm}^{-1}\right)\right] }
\end{aligned}
$$

Calibration on difference in wavenumber was not carried out in this measurement because of small difference of the wavenumber between crystalline region $\left(997 \mathrm{~cm}^{-1}\right)$ and amorphous region $\left(972 \mathrm{~cm}^{-1}\right)$ in spite of $c a .0 .66 \mu \mathrm{m}$ of the effective analytical thickness on a Ge prism. 
The measurement of bulk crystallinity from FT-IR was also carried out in this study by the $\mathrm{KBr}$ method.

\section{Surface Wettability}

Surface wettability of polypropylene films was characterized by dynamic contact angle measurement based on Wilhelmy plate technique. $^{8}$ The polypropylene films $(24 \times 50 \mathrm{~mm}$, $0.3-0.45 \mathrm{~mm}$ thickness) were used after washing with ethanol and subsequent drying in vacuo. PSt was used as a reference, which was cast on a glass plate $(24 \times 50 \mathrm{~mm}, 0.2 \mathrm{~mm}$ thickness) from a solution in toluene $\left(10 \mathrm{gl}^{-1}\right)$. Dynamic contact angle equipment (DCA-100, Orientec Co., Tokyo, Japan) was used in this study. Here, a motor drove the crosshead to advance or withdraw the water container $\left(100 \mathrm{ml}\right.$ at $\left.25^{\circ} \mathrm{C}\right)$ at a controlled speed of $20 \mathrm{~mm} \mathrm{~min}^{-1}$. The surface tension was measured with a high sensitivity strain gauge. The basic equation for calculation for contact angle is as follows:

$$
F=m g+p \gamma_{\mathrm{L}} \cos \theta-F_{\mathrm{b}}
$$

where $F$ is total force, $m$ mass of the plate, $g$ acceleration due to gravity, $p$ perimeter of the plate, $\gamma_{\mathbf{L}}$ surface tension of water, $\theta$ contact angle, and $F_{\mathrm{b}}$ buoyance force. Advancing contact angle $\left(\theta_{\mathrm{A}}\right)$ and receding contact angle $\left(\theta_{\mathbf{R}}\right)$ were calculated from the surface tension at zero displacement during immersion of the specimen in the water and withdrawal of the specimen from the water, respectively. ${ }^{9}$

\section{Plasma Protein Adsorption}

Protein adsorption onto polypropylene surfaces was measured using a quartz crystal microbalance (QCA917, Seiko EG \& G, Tokyo, Japan). ${ }^{10,11}$ A $9 \mathrm{MHz}$, AT-cut quartz crystal with gold electrodes on both sides was used as the piezoelectric resonator. The electrode area of the piezoelectric crystal resonator was $0.20 \mathrm{~cm}^{2}$. The polypropylene was cast on the quartz crystal from a solution in xylene $\left(10 \mathrm{gl}^{-1}\right)$, followed by drying in vacuo for $24 \mathrm{~h}$. PSt was used as a reference for protein adsorption, which was cast on the quartz crystal from a solution in toluene $\left(10 \mathrm{gl}^{-1}\right)$. Bovine serum albumin (BSA) and bovine plasma fibrinogen (BPF) purchased from Sigma Co., St Louis, U.S.A., were used without further purification. The polymer-coated piezoelectric crystal resonator was set in the cell, and immersed with $50 \mathrm{ml}$ of $0.1 \mathrm{M}$ phosphate buffer solution (PBS, $\mathrm{pH}$ 7.4). After the resonant frequency, resonant resistance and potential of the resonator became stable, $1 \mathrm{ml}$ protein solution in PBS was injected into the $50 \mathrm{ml}$ PBS, followed by measurement of frequency shift of the resonator due to protein adsorption. The temperature was kept at $25^{\circ} \mathrm{C}$ by a thermostatic water bath during measurement.

\section{RESULTS AND DISCUSSION}

Surface Crystallinity and Wettability of Polypropylene Films

Table I summarizes the bulk properties of polypropylene films used in this study. Variation in the semicrystalline state was observed in terms of bulk crystallinity and long period. No different tendency among PP (A1-12), EPR (C1-3), and EPB (B1-3) films is observed in Table I. No relation was also observed between bulk crystallinity and long period of PP films, as summarized in Table I. Thus, a series of these polypropylene films may have variation of crystallinity suitable for this study.

Polymeric materials are used by casting from solution or moulding from melt on a substrate. For example, one side of a polymer film is exposed to air while the other one is in contact with a substrate surface if the polymer film is cast on the substrate. Therefore, polymer surfaces in general may be different in structure from the bulk. This is due to a thermodynamical equilibrium in which polymer surfaces can be formed as to minimize interfacial free energy with air. Then, surface 
Table I. Bulk and surface properties of polypropylene films

\begin{tabular}{|c|c|c|c|c|c|c|c|}
\hline \multirow{2}{*}{ Code $^{a}$} & \multicolumn{2}{|c|}{ Crystallinity $/ \%$} & \multirow{2}{*}{$\frac{\text { Long period }^{\mathrm{d}}}{\mathrm{nm}}$} & \multicolumn{4}{|c|}{ Contact angle (degree) } \\
\hline & Bulk $^{b}$ & Surface $^{\mathfrak{c}}$ & & Advancing & Receding & Average $^{e}$ & Hysteresis $^{\mathrm{f}}$ \\
\hline Al & 70.1 & 55.8 & - & 103 & 87 & 95 & 17 \\
\hline $\mathrm{A} 2$ & 70.9 & 57.2 & - & 94 & 78 & 86 & 16 \\
\hline A3 & 59.3 & 57.5 & - & 91 & 72 & 82 & 19 \\
\hline A4 & 62.3 & 53.1 & - & 91 & 72 & 82 & 19 \\
\hline A5 & 71.9 & 68.5 & - & 83 & 66 & 74 & 17 \\
\hline A6 & 66.0 & 56.5 & - & 85 & 72 & 79 & 14 \\
\hline A7 & 63.1 & 64.0 & 14 & 96 & 72 & 84 & 24 \\
\hline A 8 & 60.0 & 53.4 & 14 & 96 & 74 & 85 & 22 \\
\hline A9 & 61.5 & 59.4 & 13 & 94 & 69 & 82 & 25 \\
\hline A 10 & 63.4 & 64.0 & 16 & 96 & 70 & 83 & 26 \\
\hline A11 & 63.8 & 66.7 & 12 & 96 & 62 & 79 & 34 \\
\hline A. 12 & 62.1 & 50.7 & 15 & 101 & 74 & 87 & 27 \\
\hline B1 & 51.2 & 41.2 & - & 107 & 91 & 99 & 16 \\
\hline B2 & 56.1 & 43.5 & - & 103 & 84 & 94 & 19 \\
\hline B3 & 58.1 & 48.5 & - & 99 & 84 & 92 & 15 \\
\hline $\mathrm{Cl}$ & 58.2 & 53.7 & - & 104 & 87 & 95 & 17 \\
\hline $\mathrm{C} 2$ & 53.4 & 47.3 & - & 104 & 91 & 97 & 13 \\
\hline C3 & 49.1 & 42.4 & - & 107 & 88 & 97 & 19 \\
\hline
\end{tabular}

${ }^{a}$ A, homopolymer; B, block-type copolymer with ethylene; C, random copolymer with ethylene. ${ }^{b}$ Bulk crystallinity was determined by FT-IR measurements. ${ }^{\mathrm{c}}$ Surface crystallinity was determined by ATR-FT-IR measurements. ${ }^{\mathrm{d}}$ Long period was determined by SAXS measurements. ${ }^{\mathrm{e}}$ Average $=($ advancing contact angle + receding contact angle) $/ 2 .{ }^{\mathrm{f}}$ Hysteresis $=$ advancing contact angle - receding contact angle.

properties of polypropylene films were investigated by ATR-FT-IR and dynamic contact angle measurements, the results of which were also summarized in Table I. The results of the ATR-FT-IR measurements revealed that the surface crystallinity of polypropylene films was well correlated with bulk crystallinity, as shown in Figure 1. The bulk crystallinity of polypropylene films obtained by ATR-FT-IR measurements was confirmed to be consistent with those obtained by differential scanning calorimetric measurements (data not shown). There was no difference in magnitudes of contact angle between both sides of polypropylene films because both sides of these films were prepared in contact with a substrate at the melt-moulding process. The addition of stabilizer (Irganox ${ }^{\circledR} 1010$ ) to polypropylenes had no effect on the anisotropy of crystallinity in these films because enrichment of this stabilizer onto the outermost surfaces may not

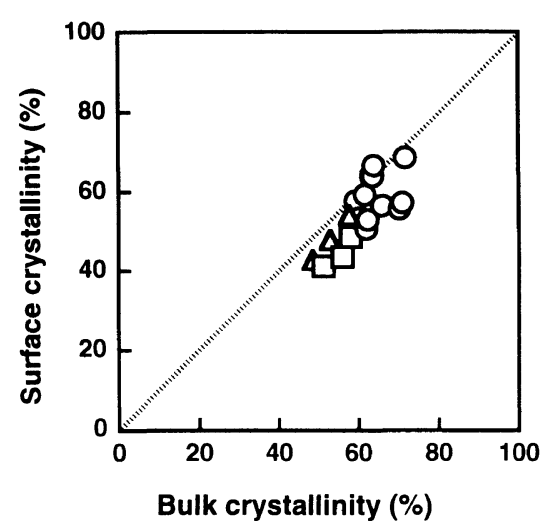

Figure 1. Relation of crystallinity to surface and bulk of polypropylene films. $\bigcirc$, PP; $\square$, EPB; $\triangle$, EPR.

occur due to a relatively larger molecular weight $(M W=1178)$ and higher melting temperature $\left(110-125^{\circ} \mathrm{C}\right)$. No difference in the relation among three types of polypropylenes, PP, EPR, and EPB, can be observed in Figure 1. The crystallization of outermost surfaces is 
N. Yur et al.

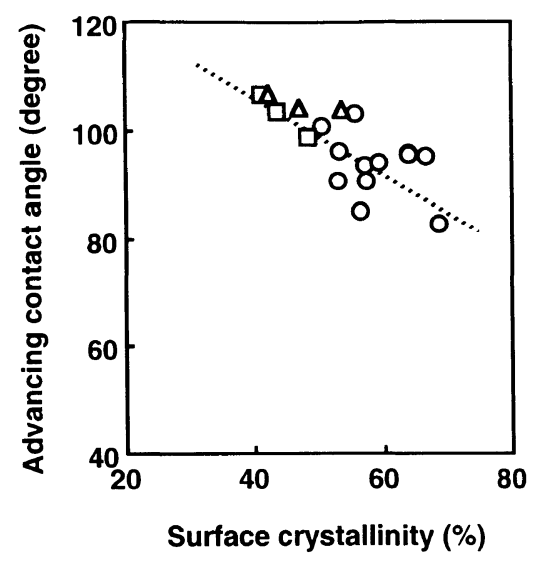

Figure 2. Relation of advancing contact angle with surface crystallinity of polypropylene films. O, PP; $\square$, $\mathrm{EPB} ; \triangle$, EPR.

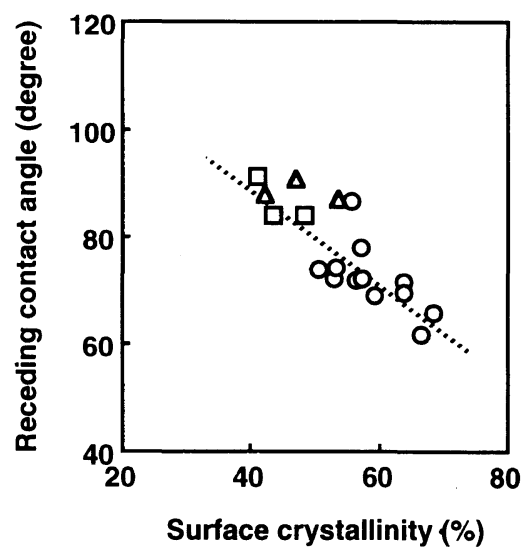

Figure 3. Relation of receding contact angle with surface crystallinity of polypropylene films. $O$, PP; $\square$, EPB; $\triangle$, EPR.

thus almost the same as that of bulk in these polypropylene films.

The surface wettability of polypropylene films was measured in order to examine the relation with the surface crystallinity, as shown in Figures 2-4. It was found that surface wettability of polypropylene films was well correlated with surface crystallinity: higher crystalline surfaces exhibited lower contact angles. This indicates that crystalline regions have higher surface free energy than amorphous regions in these polypropylene surfaces. $\theta_{\mathrm{A}}$ is dependent on the components of lower

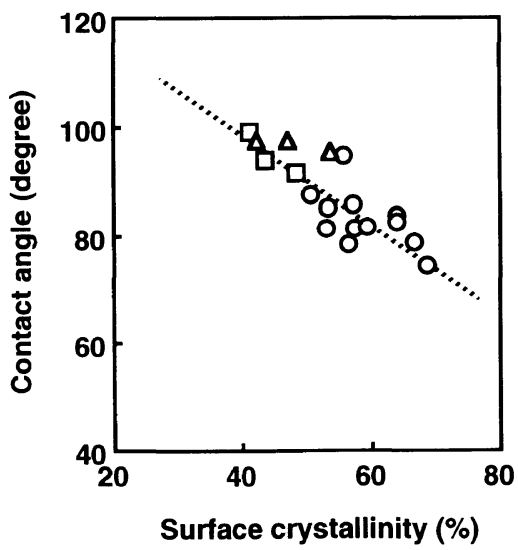

Figure 4. Relation of contact angle with surface crystallinity of polypropylene films. $\mathrm{O}, \mathrm{PP} ; \square, \mathrm{EPB} ; \triangle$, EPR.

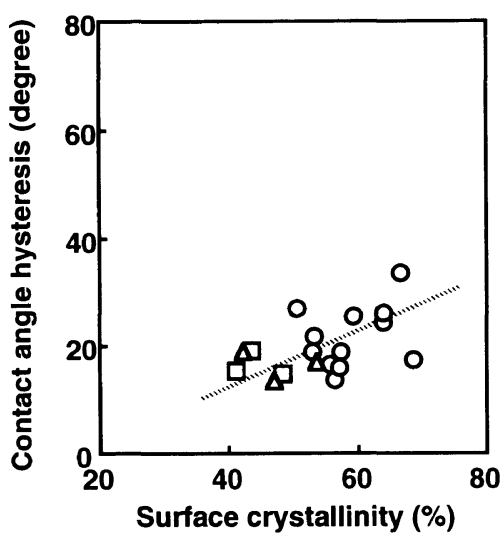

Figure 5. Relation of contact angle hysteresis with surface crystallinity of polypropylene films. $O$, PP; $\square$, $\mathrm{EPB} ; \triangle$, EPR.

surface free energy whereas $\theta_{\mathbf{R}}$ is affected by the components of higher surface free energy in dynamic contact angle measurements. ${ }^{8}$ Thus, scattering of both $\theta_{\mathrm{A}}$ values in higher crystalline surfaces (Figure 2) and $\theta_{\mathbf{R}}$ values in lower crystalline surfaces (Figure 3 ) may be caused by the reorganization of outermost surfaces of the polypropylenes with environmental change from air to water. Presumably, amorphous chains at their surfaces might have such mobilities in response to such environmental change. Higher crystallinity surfaces have a tendency of exhibit larger contact angle hysteresis, as shown in Figure 5. This may 
indicate that environmental response of polypropylene surfaces is controlled by terminal moieties and/or tie molecules of amorphous chains between crystalline regions rather than amorphous regions themselves.

\section{Plasma Protein Adsorption onto Polypropylene Films}

One of the first events to occur when a polymer surface contacts blood is the adsorption of blood plasma proteins onto the surface. Protein adsorption can dominate subsequent interactions of platelets with the surface leading to thrombus formation. Protein type, its secondary structure, and amount of protein in the adsorbed layer change continuously, depending on adsorption time, and in turn, they strongly affect platelet activation on polymer surfaces. ${ }^{12}$ On microdomain-structured surfaces, preferable adsorption of albumin and fibrinogen onto hydrophilic and hydrophobic microdomains, respectively, has been reported elsewhere. ${ }^{13}$ These results support the hypothesis that the control and organization of protein adsorption on microdomain-structured surfaces play an important roles in preventing surface-induced platelet adhesion and activation. ${ }^{14}$

Adsorption of BSA and BPF onto polypropylene surfaces with a variation of surface crystallinity was examined by a quartz crystal microbalance, the results of which are shown in Figures 6 and 7, respectively. Since polypropylene surfaces were prepared on a quartz crystal by cast from xylene, it should be noted that these surfaces are not the same as those of melt-moulding films. This might lead to differences in surface structures including the degree of crystallinity and wettability. However, these cast surfaces are considered to have surface structures corresponding to the melt-moulding films because the crystallinity of these films were varied by changing polymerization conditions and not by thermal history during the moulding and preparation processes. PSt film was also examined as a reference because this surface has been extensively studied in terms of protein adsorption. The amount of substance on a quartz crystal electrode is generally determined by the following equation.

$$
-\Delta F=k \Delta m
$$

where $\Delta F$ is the frequency shift, $\Delta m$ is the mass change on the surface of the resonator, and $K$ is a constant depending on the characteristics of the piezoelectric crystal. If $K$ is equal to $9.5 \times 10^{8} \mathrm{~Hz} \mathrm{~g}^{-1}$ mentioned in a reference, ${ }^{10}$ the amount of adsorbed protein on the surface can be calculated. However, in this study, frequency shift due to protein adsorption was directly used as the index of protein adsorption because $K$ is influenced by the nature of adsorbed and dissolved proteins: e.g., conformational-changed tight adsorption, weak adsorption with native conformation, and the molecular weights (viscosity) of dissolved proteins. It is likely that the frequency shift may show no correlation with the amount of adsorbed proteins even if multi-layered protein adsorption is occurred with increasing the concentration of protein solution. Such phenomena may cause error in the estimation of the amount of adsorbed proteins. Thus, protein solutions with relatively low concentrations were used in this study. In Figures 6 and 7, it is observed that the frequency shift due to both albumin and fibrinogen adsorptions varies dependent upon a series of polypropylene films. The concentration-dependency of both protein adsorptions may indicate the mode of protein adsorption as well as degree of protein-surface interaction. Various polypropylene surfaces were observed to show higher adsorption of albumin and fibrinogen than the PSt surface although it is limited within the range of relatively low concentration of protein solution. In our previous study, equilibrium adsorption levels of ${ }^{125} \mathrm{I}$-labeled rabbit serum albumin and rabbit plasma fibrinogen on PSt surface were 1.24 and $0.52 \mu \mathrm{g} \mathrm{cm}^{-2}$, respectively. ${ }^{3}$ Assuming $K$ is equal to $9.5 \times 10^{8} \mathrm{~Hz} / \mathrm{g}^{-1}$ 
N. YUI et al.

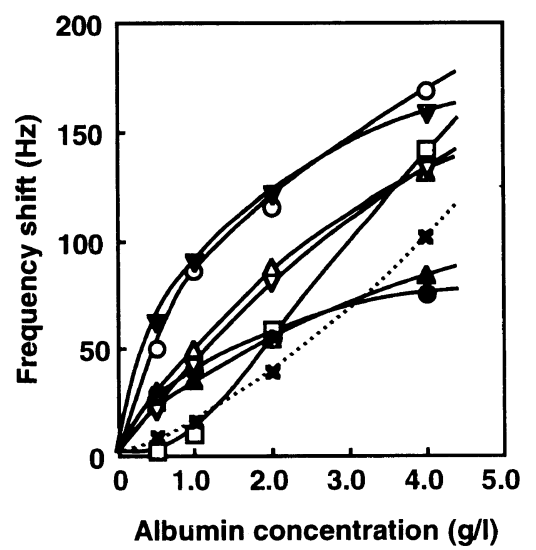

Figure 6. Albumin adsorption on polypropylene films at $25^{\circ} \mathrm{C}$ in PBS (pH 7.4). O, A11; $\triangle, \mathrm{A} 7 ; \square, \mathrm{A} 9 ; \mathrm{O}, \mathrm{A} 8$; $\mathbf{\Delta}, \mathrm{A} 12 ; \nabla, \mathrm{B} 3 ; \boldsymbol{\nabla}, \mathrm{B} 1 ; \mathbf{x}$, PSt.

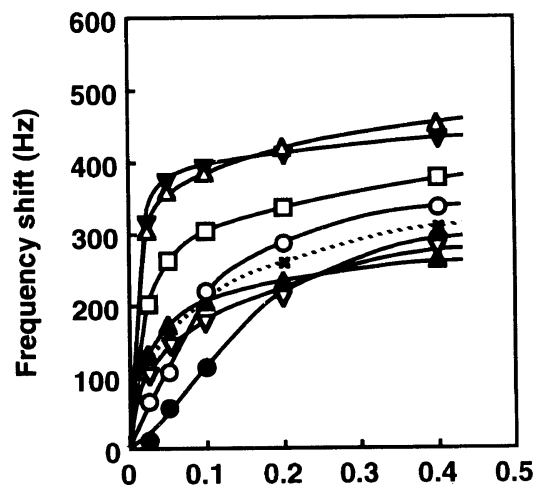

Fibrinogen concentration ( $g / /)$

Figure 7. Fibrinogen adsorption on polypropylene films at $25^{\circ} \mathrm{C}$ in PBS (pH 7.4). O, A11; $\triangle, \mathrm{A} 7 ; \square, \mathrm{A} 9 ; 0, \mathrm{~A} 8$; $\mathbf{A}, \mathrm{A} 12 ; \nabla, \mathbf{B} 3 ; \boldsymbol{\nabla}, \mathbf{B} 1 ; \boldsymbol{x}$, PSt.

as mentioned above, the calculated amounts of adsorbed albumin (concentration of RSA solution: $4.0 \mathrm{gl}^{-1}$ ) and fibrinogen (concentration of RPF solution: $0.4 \mathrm{gl}^{-1}$ ) onto PSt surface in this study were 0.53 and $1.64 \mu \mathrm{g} \mathrm{cm}^{-2}$, respectively. As seen in Figure 6, albumin adsorption onto PSt surface seems not to reach an equilibrium level. Thus, the calculated value of $0.535 \mu \mathrm{g} \mathrm{cm}^{-2}$ may be reasonable. A much higher value of fibrinogen adsorption (1.64 $\mu \mathrm{g} \mathrm{cm}^{-2}$ ) may indicate an inappropriate assumption of $K$ value because of relatively high viscosity in fibrinogen solution used in

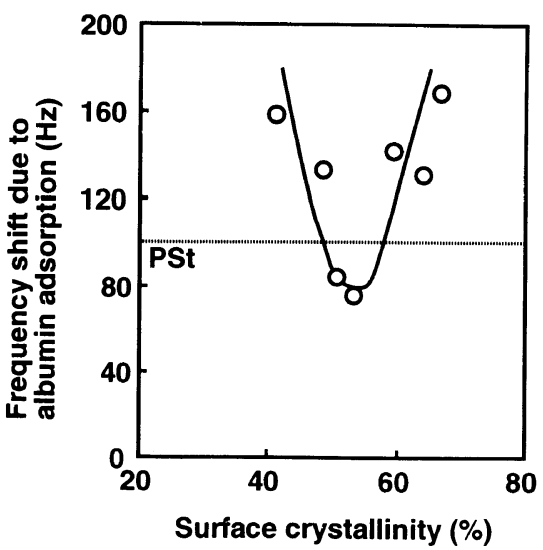

Figure 8. Relation between albumin adsorption and surface crystallinity of polypropylene films (albumin concentration: $4.0 \mathrm{gl}^{-1}$ ).

this study.

Preferable albumin adsorption onto more hydrophilic surfaces has been reported in several aspects, a representative of which was mentioned above. ${ }^{13}$ In our previous study, albumin adsorption was found to be dependent upon the crystallinity of poly(propylene oxide)-nylon 610 block-copolymer surfaces: albumin adsorption on block-copolymer surfaces decreases with increasing the amorphous poly(propylene oxide) content in the copolymer, which is explained in terms of preferable albumin adsorption onto more hydrophilic surfaces. $^{3}$ It is obvious that polypropylene surfaces used in this study have more hydrophobic character than PSt surface, since $\theta_{\mathrm{A}}$ and $\theta_{\mathrm{R}}$ of PSt surface were 88 and 68 degrees, respectively, from the dynamic contact angle measurement. Therefore, higher albumin adsorption onto polypropylene surfaces than PSt surface (Figure 6) cannot be explained by surface free energy. Furthermore, the frequency shift due to albumin adsorption was minimized at the polypropylene films with a particular surface crystallinity (ca. $50 \%$ ), as summarized in Figure 8. This indicates that surface crystallinity of polypropylene films is responsible for reduced albumin adsorption on the surfaces. This finding suggests the pos- 
sibility of regulating protein adsorption on polypropylene surfaces by controlling surface crystallinity, including the size and distribution of crystalline and amorphous regions.

Fibrinogen adsorption on polymer surfaces has been given much attention because it dominates several events concerning blood coagulation. $^{3,15}$ In particular, the thrombogenic character of adsorbed fibrinogen surface compared with albumin surface has been reported elsewhere. ${ }^{3,16}$ In our previous study, minimum fibrinogen adsorption was observed on a particular crystalline-amorphous microstructured surface which exhibited excellent blood compatibility while increased fibrinogen adsorption was observed on thrombogenic surfaces such as PSt and nylon 610 surfaces. $^{3}$ Thus, fibrinogen adsorption onto polymer surfaces can be an important index for estimating the blood compatibility of these surfaces. The frequency shift due to fibrinogen adsorption was minimized at the polypropylene films with a particular surface crystallinity ( $c a$. $50 \%$ ) although it was almost the same as that of thrombogenic PSt surface, as summarized in Figure 9. This apparently indicates that control of surface crystallinity on polypropylene films can lead to reduced fibrinogen adsorption on surfaces presumably connected with blood compatibility. From the results of Figures 8 and 9 , it is indicated that the polypropylene surfaces with a particular surface crystallinity ( $c a$. 50\%) reduced the adsorption of two types of proteins with different shapes and sizes (albumin and fibrinogen). The mechanism of minimizing the adsorption of the proteins on these polyolefin surfaces is not well understood yet. This will be considered in our next issue.

In conclusion, polypropylene films showed variation of surface crystallinity, which was well correlated with surface wettability (contact angle): crystalline regions have higher surface free energy than amorphous regions in these surfaces. The adsorption of both albumin and fibrinogen was minimized at polypropylene

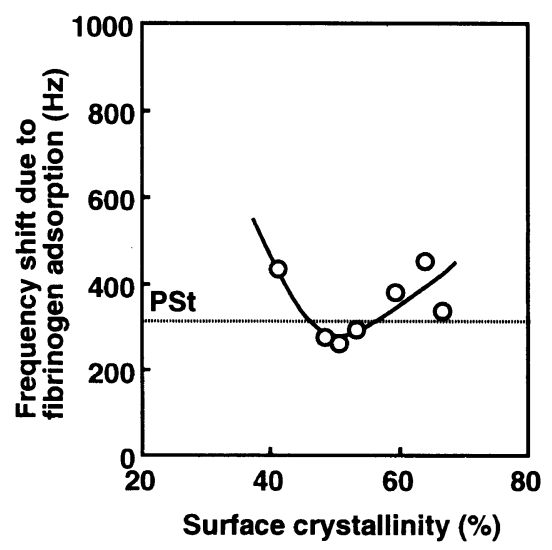

Figure 9. Relation between fibrinogen adsorption and surface crystallinity of polypropylene films (fibrinogen concentration: $0.4 \mathrm{~g}^{-1}$ ).

surfaces with a particular crystallinity, which indicates the control of surface crystallinity including the size and distribution of crystalline and amorphous regions can dominate the fate of plasma protein adsorption.

Recently, the blood compatibility of these polypropylene films was evaluated by platelet adhesion on these surfaces. ${ }^{17}$ It was observed that platelet adhesion was dependent on the surface crystallinity of these surfaces, the detailed results of which will be reported in our forthcoming paper. Therefore, it is suggested that the design of blood compatible polypropylene surfaces will be achieved through control of surface semicrystalline states.

Acknowledgments. This study was financially supported by a Grant-in-Aid from Shibuya Science-Culture-Sports Foundation, Japan. The authors are grateful to Associate Prof. Dr. Kazuhiko Ishihara, Tokyo Medical \& Dental University, for his valuable suggestion in the measurements of protein adsorption by the quartz crystal microbalance, and Chisso Co., Tokyo, Japan, for their kind supply of polypropylene films and their help in SAXS measurements. 


\section{REFERENCES}

1. N. Yui, Y. Takahashi, K. Sanui, N. Ogata, K. Kataoka, T. Okano, and Y. Sakurai, Jpn. J. Artif. Organs, 10, 1070 (1981).

2. N. Yui, K. Sanui, N. Ogata, K. Kataoka, T. Okano, and Y. Sakurai, J. Biomed. Mater. Res., 20, 929 (1986).

3. Y. G. Takei, N. Yui, T. Okano, A. Maruyama, K. Sanui, Y. Sakurai, and N. Ogata, J. Biomater. Sci. Polym. Ed., 6, 149 (1994).

4. M. Terano, M. Saito, and T. Kataoka, Makromol. Chem. Rapid Commun., 13, 103 (1992).

5. C. F. Payn, "Proceeding of Metcon '93," Catalyst Consultants Inc., Spring House, PA, 1993, p 51.

6. T. Shimizu, K. Kouketsu, Y. Morishima, S. Goto, I. Hasegawa, T. Kamiya, Y. Tamura, and S. Kora, Transfusion, 29, 292 (1989).

7. R. G. Quynn, J. L. Riley, D. A. Young, and H. D. Noether, J. Appl. Polym. Sci., 2, 166 (1959).

8. K. G. Tingey, J. D. Andrade, C. W. McGary, Jr., and R. J. Adrahala, "Polymer Surface Dynamics", J. D. Andrade, Ed., Plenum, New York, 1988, p 153.

9. T. Teraya, A. Takahara, and T. Kajiyama, Polymer, 31, 1149 (1990).

10. X. Ye, H. Muramatsu, K. Kimura, T. Sakuhara, and T. Ataka, J. Electroanal. Chem., 314, 279 (1991).

11. S. Tanaka, Y. Iwasaki, K. Ishihara, and N. Nakabayashi, Macromol. Rapid Commun., 15, 319 (1994).

12. J. L. Brash and P. ten Hove, Thromb. Haemostas., 51, 326 (1984).

13. T. Okano, S. Nishiyama, I. Shinohara, T. Akaike, and Y. Sakurai, Polym. J., 10, 223 (1978).

14. C. Nojiri, T. Okano, H. A. Jacobs, K. D. Park, S. F. Mohammad, D. B. Olsen, and S. W. Kim, J. Biomed. Mater. Res., 24, 1151 (1990).

15. J. L. Brash, Ann. N. Y. Acad. Sci., 516, 206 (1987).

16. E. W. Salzman, J. Lindon, G. McManama, and J. A. Ware, Ann. N. Y. Acad. Sci., 516, 184 (1987).

17. N. Yui, Y. Suzuki, H. Mori, and M. Terano, in preparation. 